\title{
Delirium Associations with Antibiotics: A Pharmacovigilance Study of the FDA Adverse Event Reporting System (FAERS)
}

\author{
Chengwen Teng ${ }^{1}\left[\right.$ (D) Christopher R. Frei ${ }^{2,3,4,5}$
}

Accepted: 22 June 2021 / Published online: 17 July 2021

(c) The Author(s) 2021

\begin{abstract}
Background Ertapenem, cefepime, imipenem, ofloxacin, ceftazidime, clarithromycin, cefaclor, levofloxacin, linezolid, moxifloxacin, azithromycin, piperacillin-tazobactam, trimethoprim-sulfamethoxazole, metronidazole, ciprofloxacin, and cefuroxime are known to be associated with delirium. Other antibiotics may also lead to delirium, but no study has systemically compared delirium associations for many available antibiotics.

Objective The objective of this study was to evaluate the association between delirium and antibiotics using the FDA Adverse Event Reporting System (FAERS).

Methods FAERS reports from January 1, 2004 to December 31, 2018 were included in the study. Reporting odds ratios (RORs) and corresponding 95\% confidence intervals (95\% CI) for the association between antibiotics and delirium were calculated. An association was considered to be statistically significant when the lower limit of the $95 \%$ CI was greater than 1.0 . Results A total of 10,015,622 reports (including 16,982 delirium reports) were considered, after inclusion criteria were applied. Statistically significant delirium RORs (95\% CI) for antibiotics were: ertapenem 21.07 (16.38-27.10), cefepime 9.8 (6.37-15.09), imipenem 9.68 (6.75-13.89), ofloxacin 7.73 (4.00-14.92), ceftazidime 6.09 (2.73-13.62), clarithromycin 5.34 (4.37-6.53), cefaclor 5.32 (1.71-16.58), ampicillin-sulbactam 4.49 (2.13-9.45), levofloxacin 4.47 (3.88-5.16), linezolid 4.33 (3.28-5.72), moxifloxacin 3.51 (2.81-4.38), azithromycin 2.76 (2.09-3.64), piperacillin-tazobactam 2.41 (1.47-3.93), trimethoprim-sulfamethoxazole 2.36 (1.61-3.47), metronidazole 1.85 (1.31-2.60), ciprofloxacin 1.83 (1.44-2.33), and cefuroxime 1.81 (1.03-3.20).

Conclusion This study found statistically significant increased risk of reporting delirium with ertapenem, cefepime, imipenem, ofloxacin, ceftazidime, clarithromycin, cefaclor, ampicillin-sulbactam, levofloxacin, linezolid, moxifloxacin, azithromycin, piperacillin-tazobactam, trimethoprim-sulfamethoxazole, metronidazole, ciprofloxacin, and cefuroxime.
\end{abstract}

Chengwen Teng

TENGC@mailbox.sc.edu

1 Department of Clinical Pharmacy and Outcomes Sciences, College of Pharmacy, The University of South Carolina, 715 Sumter Street, Columbia, SC 29208, USA

2 Pharmacotherapy Division, College of Pharmacy, The University of Texas at Austin, San Antonio, TX, USA

3 Pharmacotherapy Education and Research Center, Long School of Medicine, The University of Texas Health Science Center at San Antonio, San Antonio, TX, USA

4 South Texas Veterans Health Care System, San Antonio, TX, USA

5 University Health System, San Antonio, TX, USA

\section{Key Points}

This study identifies previously unknown delirium reporting association with ampicillin-sulbactam.

Ertapenem had the highest delirium reporting association among antibiotics evaluated in the study.

Older patients had a higher delirium Reporting Odds Ratio than younger patients among most of the antibiotic classes analyzed. 


\section{Introduction}

Delirium is diagnosed in the presence of a disturbance in attention and awareness that develops over a short period of time, represents a change from baseline, tends to fluctuate, has an additional cognitive disturbance (e.g., memory deficit, disorientation, language, visuospatial ability, or perception), all of which are not better explained by other diagnoses (e.g., coma) [1]. Delirium is associated with higher mortality rates, affects up to $50 \%$ of hospitalized elderly patients, and costs more than $\$ 164$ billion per year in the United States [2]. Delirium may be nothing more than a marker of severity of illness likened to a cough for pneumonia. Treatment of pneumonia resolves the cough like treatment of sepsis may resolve delirium. This point was proven in multiple trials of critically ill patients including BRAIN-ICU, which showed ICU delirium is associated with long-term cognitive impairment, and MIND-USA, which showed delirium treatment in those with hypoactive delirium does not improve outcome [3, 4].

Many antibiotics are known to be associated with delirium. Overall quality of evidence for many reports is very low. Multiple case reports have reported on ertapeneminduced delirium [5-7]. A case of hypoactive delirium and another case of encephalopthy as adverse effects of imipenem were reported $[8,9]$. A case report demonstrated a case of delirium with hallucinations likely caused by a combination of diphenhydramine and linezolid [10]. Delirium was also reported to be induced by clarithromycin monotherapy or a combination of clarithromycin and fluoxetine [11-13]. Delirium was associated with azithromycin in two geriatric patients treated for lower respiratory tract infection [14]. There are at least 6 published case reports of delirium associated with the use of azithromycin, including one pediatric patient, and 12 published case reports of delirium associated with the use of clarithromycin [15]. Four fluoroquinolone antibiotics, which were ofloxacin, levofloxacin, moxifloxacin, and ciprofloxacin, were all reported to be associated with delirium [16-19]. Cefepime use was associated with increased likelihood and duration of acute encephalopathy (presence of delirium or depressed level of consciousness in the absence of deep sedation) in critically ill patients in a retrospective case-control study [20]. A nested cohort study concluded that first, second, and third-generation cephalosporins doubled the odds of delirium [21]. A case report reported higher-level gait disorder and nocturnal delirium likely induced by trimethoprim-sulfamethoxazole [22]. In another case report, metronidazole and levofloxacin induced delirium in a chronic kidney disease patient [23]. Delirium was also reported to be induced by piperacillin-tazobactam in a peritoneal dialysis patient [24].
Few studies have looked at antibiotics and compared their associations with delirium. The objective of this study was to evaluate the association between delirium and antibiotics using FDA Adverse Event Reporting System (FAERS).

\section{Methods}

\subsection{Data Source}

FAERS is a publicly available database composed of adverse event reports that were submitted to United States Food and Drug Administration (FDA) [25]. FAERS data contain drug information (drug name, active ingredient, route of administration, the drug's reported role in the event) and reaction information. Healthcare professionals, consumers or manufacturers may submit reports. The types of data found in FAERS include reason for administration, specific reaction, seriousness, outcome, gender, event data, patient age, who sent the report, reporter type, country, whether or not it was reported to the manufacturer and whether or not it was published. Quarterly Data Files were used to obtain FAERS data. The Institutional Review Board (IRB) of The University of South Carolina determined that this study is not human subjects research (IRB number: Pro00101342).

\subsection{Study Design}

FAERS data from January 1, 2004 to December 31, 2018 were included in the study. Some adverse event reports were submitted multiple times with updated information. Therefore, duplicate reports were removed by case number, with the most recent submission included in the study.

\subsection{Drug Exposure Definition}

Each antibiotic was identified in FAERS by generic and brand names listed in the Drugs@FDA Database [26]. Antibiotics with less than three delirium Adverse Drug Reaction (ADR) reports were excluded from this data analysis [27]. All reports were included, regardless of route of administration and the drug's reported role in the event. Antibiotics included in the study were ertapenem, imipenem, meropenem, linezolid, clarithromycin, azithromycin, erythromycin, ofloxacin, levofloxacin, moxifloxacin, ciprofloxacin, cefepime, ceftazidime, cefaclor, cefuroxime, cephalexin, ceftriaxone, trimethoprim-sulfamethoxazole, metronidazole, ampicillin-sulbactam, piperacillin-tazobactam, amoxicillin-clavulanate, amoxicillin, nitrofurantoin, daptomycin, vancomycin, minocycline, and clindamycin. Antibiotic classes included in the study were carbapenems, macrolides, fluoroquinolones, cephalosporins, and penicillin combinations. 


\subsection{Adverse Drug Reaction Definition}

FAERS defines ADRs using Preferred Terms (PT) from the Medical Dictionary for Regulatory Activities (MedDRA) [28]. Preferred Term "Delirium" was used to identify delirium cases.

\subsection{Statistical Analysis}

A disproportionality analysis was conducted by computing Reporting Odds Ratios (ROR) and corresponding 95\% confidence intervals $(95 \% \mathrm{CI})$ for the association between delirium and each antibiotic class or individual antibiotic [29]. ROR was calculated as the ratio of the odds of reporting delirium versus all other ADRs for a given drug, compared with this reporting odds for all other drugs present in FAERS [29]. An association was considered to be statistically significant if the lower limit of $95 \%$ CI was above 1.0 [29]. A subgroup analysis was performed on patients who were 65 years or older and patients less than 65 years old. Data analysis was performed using Microsoft Excel 2016 (Microsoft Corporation, Redmond, WA) and SAS 9.4 (SAS Institute, Cary, NC).

\section{Results}

A total of 10,015,622 reports (including 16,982 delirium reports) were considered, after inclusion criteria were applied (Fig. 1). The number of cases for each drug and drug class is listed in Fig. 2. Statistically significant delirium RORs (95\% CI) for antibiotic classes were: carbapenems 10.49 (8.59-12.80), macrolides 3.65 (3.11-4.28), fluoroquinolones 3.40 (3.05-3.78), cephalosporins 2.54 (1.99-3.23), and penicillin combinations $1.62(1.20-2.18)$.

Statistically significant delirium RORs $(95 \% \mathrm{CI})$ for antibiotics were: ertapenem 21.07 (16.38-27.10), cefepime 9.8 (6.37-15.09), imipenem 9.68 (6.75-13.89), ofloxacin 7.73 (4.00-14.92), ceftazidime 6.09 (2.73-13.62), clarithromycin

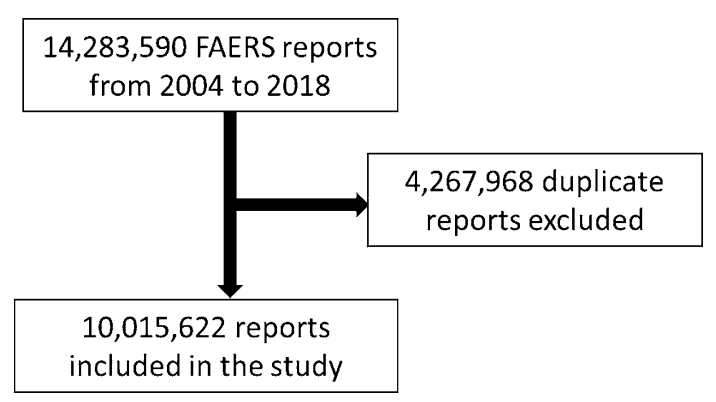

Fig. 1 Flow diagram for inclusion and exclusion of the FAERS reports
5.34 (4.37-6.53), cefaclor 5.32 (1.71-16.58), ampicillin-sulbactam 4.49 (2.13-9.45), levofloxacin 4.47 (3.88-5.16), linezolid 4.33 (3.28-5.72), moxifloxacin 3.51 (2.81-4.38), azithromycin 2.76 (2.09-3.64), piperacillin-tazobactam 2.41 (1.47-3.93), trimethoprim-sulfamethoxazole 2.36 (1.61-3.47), metronidazole 1.85 (1.31-2.60), ciprofloxacin 1.83 (1.44-2.33), and cefuroxime 1.81 (1.03-3.20) (Fig. 2).

Among patients who took carbapenems, linezolid, macrolides, fluoroquinolones, cephalosporins, trimethoprim-sulfamethoxazole, metronidazole, penicillin combinations, and clindamycin, patients who were 65 years or older had a higher delirium ROR than those less than 65 years old (Fig. 3). Among patients who took amoxicillin and vancomycin, patients who were 65 years or older had a lower delirium ROR than those less than 65 years old (RORs were not statistically significant considering that the lower limit of $95 \%$ CI was below 1.0).

\section{Discussion}

Our results indicated significant delirium reporting associations (from strongest to weakest) with the following antibiotic classes: carbapenems, macrolides, fluoroquinolones, cephalosporins, and penicillin combinations (Fig. 2).

Our results demonstrated significant delirium reporting associations (from strongest to weakest) with the following antibiotics: ertapenem, cefepime, imipenem, ofloxacin, ceftazidime, clarithromycin, cefaclor, ampicillin-sulbactam, levofloxacin, linezolid, moxifloxacin, azithromycin, piperacillin-tazobactam, trimethoprim-sulfamethoxazole, metronidazole, ciprofloxacin, and cefuroxime (Fig. 2). Our results were in agreement with previously known delirium associations with ertapenem, cefepime, imipenem, ofloxacin, ceftazidime, clarithromycin, cefaclor, levofloxacin, linezolid, moxifloxacin, azithromycin, piperacillin-tazobactam, trimethoprim-sulfamethoxazole, metronidazole, ciprofloxacin, and cefuroxime [5-24]. Ampicillin-sulbactam was found to be associated with delirium in FAERS, which was not reported in the literature.

Cefepime is known to be able to induce neurotoxicity due to its ability to cross the blood-brain barrier and to exhibit concentration-dependent competitive gamma-aminobutyric acid (GABA) antagonism [30-32]. The signs and symptoms of cefepime-induced neurotoxicity included altered mental status, reduced consciousness, confusion, myoclonus, aphasia, agitation, and seizures [32]. Many of the signs and symptoms of cefepime-induced neurotoxicity correlated well with signs and symptoms of delirium [1]. Therefore, it is not surprising to find the significant association between cefepime and delirium in FAERS.

Carbapenem antibiotics may have a higher neurotoxic potential than that of penicillins and cephalosporins [33]. 


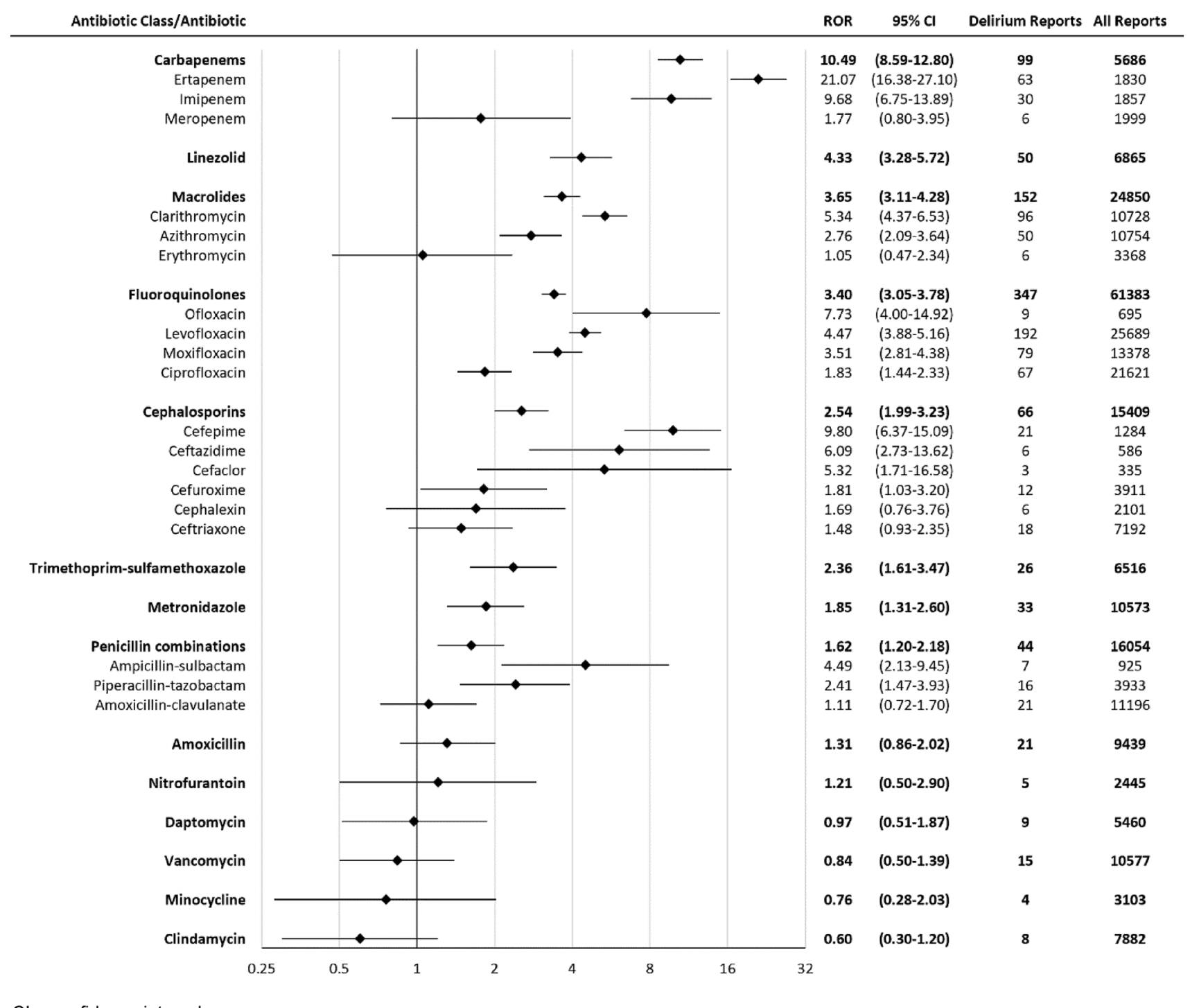

$\mathrm{Cl}=$ confidence interval

Fig. 2 Reporting odds ratios (ROR) for delirium with antibiotics

Indeed, in our study, carbapenem antibiotic class had the highest delirium ROR among all antibiotic classes included in the study (Fig. 1). This may not be a class-wide effect and certain carbapenems may be more offensive (e.g., imipenem) than others (e.g., meropenem). Prior studies have suggested that cefepime may have a tenfold greater risk for neurotoxicity than meropenem [34, 35]. Neurotoxicity of carbapenems was also thought to be caused by an interaction with GABA receptors [33]. The association between imipenem and delirium found in FAERS may be due to this mechanism.

Although ampicillin-sulbactam-induced delirium was not reported in the literature, ampicillin-sulbactam is a beta-lactam, which has the potential to induce neurotoxicity via interactions with GABA receptors [31]. The association between ampicillin-sulbactam and delirium found in
FAERS may be explained by this mechanism. It is interesting that ampicillin-sulbactam was associated with delirium given its minimal penetration across the blood-brain-barrier. Prior reports have stated its penetrance is $1 \%$ of the plasma concentration and only increases in the setting of inflamed meninges [36].

Although a case of meropenem-associated delirium was reported in the literature, our study did not find statistically significant ROR for meropenem and delirium. The low number of meropenem delirium reports in FAERS may be the reason of statistical insignificance (Fig. 2).

In the subgroup analysis, the delirium ROR rank order in both subgroups ( $<65$ years old and $\geq 65$ years old) were similar to that in all patients. Our results showed that older patients had a higher delirium ROR than younger patients 


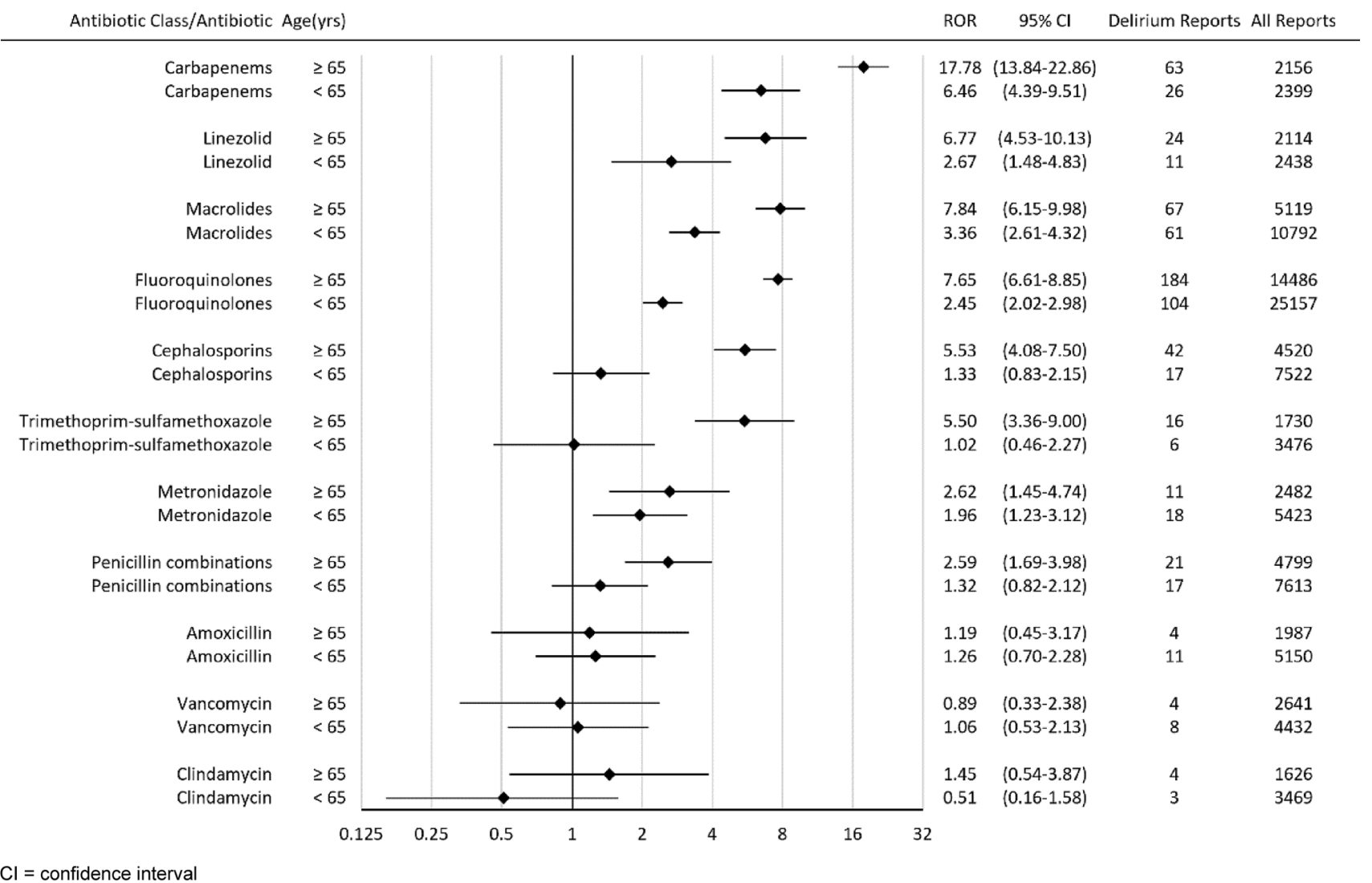

Fig. 3 Reporting odds ratios (RORs) for delirium with antibiotics stratified by age

among most of the antibiotic classes analyzed (Fig. 3). Carbapenems, macrolides, fluoroquinolones, cephalosporins, and trimethoprim-sulfamethoxazole had significantly greater associations with delirium in older patients than those in younger patients. It is known that delirium risk is higher in older patients [1]. To note, ciprofloxacin and trimethoprim-sulfamethoxazole were also included in the American Geriatrics Society Beers Criteria for potentially inappropriate medication use in older adults [37]. Ciprofloxacin was in the Beers Criteria for tendon rupture and hyperkalemia, but not for delirium.

\subsection{Limitations}

A causal relationship between a drug and an ADR cannot be determined by FAERS. Significant bias may occur because of the spontaneous and voluntary reporting of ADRs. Media attention and recent publication of an ADR in the literature might affect the reporting behaviors. The association between a drug and an ADR is confounded by comorbidities and concomitant drugs. According to FDA, information submitted has not been verified by a medical professional. FAERS data may be submitted by healthcare professionals, consumers and manufacturers. Source of submission must be considered. Some information is missing or incomplete in FAERS. There have been cases in which age was not reported or drug names were misspelled. FDA does not receive reports for every adverse event or medication error that occurs with a product. In addition, ROR only investigates an increased risk of ADR reporting and not risk of ADR occurrence in absolute terms [38]. Some of the confidence intervals are quite wide such as cefaclor $5.32(1.71-16.58)$ because of the low number of cefaclor ROR reports for delirium. An additional limitation of this study is the reliance on "delirium" as the search term in the FAERS database. Terms such as delirium, encephalopathy, acute confusional state, acute brain dysfunction and acute brain failure are often used synonymously and inappropriately in the literature [39]. Without the ability to determine how delirium was diagnosed, it is possible that numerous cases were misclassified and could have altered the findings. Despite the limitations, FAERS has a large sample size and is suitable for discovering a signal of new and rare drug-ADR associations. 


\section{Conclusion}

This study found statistically significant increased risk of reporting delirium with ertapenem, cefepime, imipenem, ofloxacin, ceftazidime, clarithromycin, cefaclor, ampicillin-sulbactam, levofloxacin, linezolid, moxifloxacin, azithromycin, piperacillin-tazobactam, trimethoprim-sulfamethoxazole, metronidazole, ciprofloxacin, and cefuroxime. Results obtained from FAERS should be interpreted with caution in the context of data limitations. Antibiotic stewardship is needed to prevent delirium and to improve health outcomes.

Acknowledgements No funding was sought for this research study. Dr. Frei was supported, in part, by a NIH Clinical and Translational Science Award (National Center for Advancing Translational Sciences, UL1 TR001120 and UL1 TR002645) while the study was being conducted. The funding sources had no role in the design and conduct of the study; collection, management, analysis, and interpretation of the data; preparation, review, or approval of the manuscript; and decision to submit the manuscript for publication. The views expressed in this article are those of the authors and do not necessarily represent the views of the Department of Veterans Affairs, the National Institutes of Health, or the authors' affiliated institutions.

\section{Declarations}

Funding No funding was sought for this research study. Dr. Frei was supported, in part, by a NIH Clinical and Translational Science Award (National Center for Advancing Translational Sciences, UL1 TR001120 and UL1 TR002645) while the study was being conducted. The funding sources had no role in the design and conduct of the study; collection, management, analysis, and interpretation of the data; preparation, review, or approval of the manuscript; and decision to submit the manuscript for publication.

Conflict of interest Dr. Frei's institution has received grants for research from AstraZeneca Pharmaceuticals in the last 36 months.

Ethics approval The Institutional Review Board (IRB) of The University of South Carolina determined that this study is not human subjects research (IRB number: Pro00101342).

Consent to participate Not applicable.

Consent for publication Not applicable.

Availability of data and material The datasets generated during and/or analysed during the current study are available from the corresponding author on reasonable request.

Code availability Codes used during the current study are available from the corresponding author on reasonable request.

Author contributions Study concept and design: all authors. Statistical analysis: CT. Interpretation of data: all authors. Drafting of the manuscript: CT. Critical revision of the manuscript for important intellectual content: all authors. Study supervision: CT.

Open Access This article is licensed under a Creative Commons Attribution-NonCommercial 4.0 International License, which permits any non-commercial use, sharing, adaptation, distribution and reproduction in any medium or format, as long as you give appropriate credit to the original author(s) and the source, provide a link to the Creative Commons licence, and indicate if changes were made. The images or other third party material in this article are included in the article's Creative Commons licence, unless indicated otherwise in a credit line to the material. If material is not included in the article's Creative Commons licence and your intended use is not permitted by statutory regulation or exceeds the permitted use, you will need to obtain permission directly from the copyright holder. To view a copy of this licence, visit http://creativecommons.org/licenses/by-nc/4.0/.

\section{References}

1. American Psychiatric Association. Diagnostic and statistical manual of mental disorders. 5th ed. Arlington: American Psychiatric Association; 2013.

2. Inouye SK, Westendorp RG, Saczynski JS. Delirium in elderly people. Lancet. 2014;383(9920):911-22.

3. Pandharipande PP, Girard TD, Jackson JC, Morandi A, Thompson JL, Pun BT, Brummel NE, Hughes CG, Vasilevskis EE, Shintani AK, Moons KG. Long-term cognitive impairment after critical illness. N Engl J Med. 2013;369(14):1306-16.

4. Girard TD, Exline MC, Carson SS, Hough CL, Rock P, Gong MN, Douglas IS, Malhotra A, Owens RL, Feinstein DJ, Khan B. Haloperidol and ziprasidone for treatment of delirium in critical illness. N Engl J Med. 2018;379(26):2506-16.

5. Veillette JJ, Van Epps P. Ertapenem-induced hallucinations and delirium in an elderly patient. Consult Pharm. 2016;31(4):207-14.

6. Apodaca K, Baker J, Bin-Bilal H, Raskin Y, Quinn DK. Ertapenem-induced delirium: a case report and literature review. Psychosomatics. 2015;56(5):561-6.

7. Lin H, Chew ST. Status epilepticus and delirium associated with ertapenem in a very elderly patient with chronic kidney disease and silent ischaemic cerebrovascular disease. Drug Saf Case Rep. 2015;2(1):19.

8. Africa BL, Carthen D, Arabelo H. A4 Delirious because of treatment: an adverse effect of imipenem. J Am Geriatr Soc. 2012;60:S18.

9. Fernández-Torre JL, Velasco M, Gutiérrez R, Fernández-Sampedro M. Encephalopathy secondary to imipenem therapy. Clin EEG Neurosci. 2004;35(2):100-3.

10. Serio RN. Acute delirium associated with combined diphenhydramine and linezolid use. Ann Pharmacother. 2004;38(1):62-5.

11. Ozsoylar G, Sayin A, Bolay H. Clarithromycin monotherapyinduced delirium. J Antimicrob Chemother. 2007;59(2):331.

12. Mermelstein HT. Clarithromycin-induced delirium in a general hospital. Psychosomatics. 1998;39(6):540-2.

13. Pollak PT, Sketris IS, MacKenzie SL, Hewlett TJ. Delirium probably induced by clarithromycin in a patient receiving fluoxetine. Ann Pharmacother. 1995;29(5):486-8.

14. Cone LA, Padilla L, Potts BE. Delirium in the elderly resulting from azithromycin therapy. Surg Neurol. 2003;59(6):509-11.

15. Pejčić AV. Delirium associated with the use of macrolide antibiotics: a review. Int J Psychiatry Clin Pract. 2020;1-14. https://doi. org/10.1080/13651501.2020.1828933

16. Odeh M, Kogan Y, Paz A, Elias N. Delirium induced by levofloxacin. J Clin Neurosci. 2019;66:262-4.

17. Tasleem H, Viswanathan R. Moxifloxacin-induced delirium with hallucinations. Psychosomatics. 2011;52(5):472-4.

18. Jay GT, Fitzgerald JM. Ciprofloxacin-induced delirium. Ann Pharmacother. 1997;31(2):252.

19. Fennig S, Mauas L. Ofloxacin-induced delirium. J Clin Psychiatry. 1992;53(4):137-8. 
20. Singh TD, O'Horo JC, Day CN, Mandrekar J, Rabinstein AA. Cefepime is associated with acute encephalopathy in critically ill patients: a retrospective case-control study. Neurocrit Care. 2020;33:695-700.

21. Grahl JJ, Stollings JL, Rakhit S, Person AK, Wang L, Thompson $\mathrm{JL}$, et al. Antimicrobial exposure and the risk of delirium in critically ill patients. Crit Care. 2018;22(1):337.

22. Dakin LE. Probable trimethoprim/sulfamethoxazole-induced higher-level gait disorder and nocturnal delirium in an elderly man. Ann Pharmacother. 2009;43(1):129-33.

23. Velickovic-Radovanovic R, Catic-Dordevic A, Dinic K, Radivojevic J, Zikic O, Cvetkovic T, et al. Metronidazole- and levofloxacin-induced psychotic disorders in chronic kidney patient. Eur J Hosp Pharm. 2019;26(6):347-9.

24. Tong MK, Siu YP, Yung CY, Kwan TH. Piperacillin/tazobactaminduced acute delirium in a peritoneal dialysis patient. Nephrol Dial Transplant. 2004;19(5):1341.

25. Food and Drug Administration. FDA Adverse Event Reporting System (FAERS). 2020. https://www.fda.gov/drugs/surveillan ce/questions-and-answers-fdas-adverse-event-reporting-systemfaers. Accessed 1 Oct 2020.

26. Food and Drug Administration. Drugs@FDA: FDA Approved Drug Products. 2020. https://www.accessdata.fda.gov/scripts/ cder/daf/index.cfm. Accessed 1 Oct 2020.

27. Evans SJ, Waller PC, Davis S. Use of proportional reporting ratios (PRRs) for signal generation from spontaneous adverse drug reaction reports. Pharmacoepidemiol Drug Saf. 2001;10(6):483-6.

28. MedDRA MSSO. Introductory Guide for Standardised MedDRA Queries (SMQs) Version 23.1. 2020. https://admin.new.meddra. org/sites/default/files/guidance/file/SMQ_intguide_23_1_English. pdf. Accessed 1 Oct 2020.

29. Bate A, Evans SJ. Quantitative signal detection using spontaneous ADR reporting. Pharmacoepidemiol Drug Saf. 2009;18(6):427-36.

30. Fugate JE, Kalimullah EA, Hocker SE, Clark SL, Wijdicks EF, Rabinstein AA. Cefepime neurotoxicity in the intensive care unit: a cause of severe, underappreciated encephalopathy. Crit Care. 2013;17(6):1-6.

31. Grill MF, Maganti R. Cephalosporin-induced neurotoxicity: clinical manifestations, potential pathogenic mechanisms, and the role of electroencephalographic monitoring. Ann Pharmacother. 2008;42(12):1843-50.

32. Payne LE, Gagnon DJ, Riker RR, Seder DB, Glisic EK, Morris JG, et al. Cefepime-induced neurotoxicity: a systematic review. Crit Care. 2017;21(1):276.

33. Norrby SR. Neurotoxicity of carbapenem antibacterials. Drug Saf. 1996;15(2):87-90.

34. Tanaka A, Takechi K, Watanabe S, Tanaka M, Suemaru K, Araki H. Comparison of the prevalence of convulsions associated with the use of cefepime and meropenem. Int J Clin Pharm. 2013;35(5):683-7.

35. Chaïbi K, Chaussard M, Soussi S, Lafaurie M, Legrand M. Not all $\beta$-lactams are equal regarding neurotoxicity. Crit Care. 2016;20(1):1-2.

36. Kearney BP, Aweeka FT. The penetration of anti-infectives into the central nervous system. Neurol Clin. 1999;17(4):883-900.

37. BY the American Geriatrics Society Beers Criteria Update Expert Panel. American Geriatrics Society 2019 Updated AGS Beers Criteria(R) for Potentially Inappropriate Medication Use in Older Adults. J Am Geriatr Soc. 2019;67(4):674-94.

38. Montastruc JL, Sommet A, Bagheri H, Lapeyre-Mestre M. Benefits and strengths of the disproportionality analysis for identification of adverse drug reactions in a pharmacovigilance database. Br J Clin Pharmacol. 2011;72(6):905-8.

39. Slooter AJ, Otte WM, Devlin JW, Arora RC, Bleck TP, Claassen J, Duprey MS, Ely EW, Kaplan PW, Latronico N, Morandi A. Updated nomenclature of delirium and acute encephalopathy: statement of ten Societies. Intensive Care Med. 2020;46(5):1020-2. 